\title{
On the homological classification of pomonoids: atomic posemilattices
}

\begin{abstract}
MATI KILP
ABSTRACT. Between different and relatively well investigated so-called flatness properties of $S$-posets there is a property called property $\left(\mathrm{P}_{w}\right)$ which, so far, has not received much attention. In a recent paper by the author pomonoids from a subclass of completely simple semigroups with adjoined identity, all of whose cyclic (Rees factor) $S$-posets satisfy property $\left(\mathrm{P}_{w}\right)$, were characterized. In this paper pomonoids which as monoids are atomic semilattices, all of whose Rees factor $S$-posets satisfy property $\left(\mathrm{P}_{w}\right)$, are characterized.
\end{abstract}

\section{Preliminaries}

Throughout this paper, $S$ will stand for a pomonoid with identity element 1 which as a monoid is an atomic semilattice with the highest element 1 and the lowest element 0 .

Recall that in a partially ordered set with the lowest element 0 , an atom is an element that covers 0, i.e., an element that is minimal among the non-zero elements. A partially ordered set with the lowest element is called atomic if every non-zero element is an atom or has an atom below it. It is clear that finite semilattices are atomic.

Recall also that if $S$ is a pomonoid, then a nonempty poset $(A, \leq)$ together with a mapping $A \times S \rightarrow A,(a, s) \mapsto a s$ is called a right $S$-poset (and the notation $A_{S}$ is used) if (1) $a\left(s s^{\prime}\right)=(a s) s^{\prime}$, (2) $a 1=a$, (3) $a \leq b$ implies $a s \leq b s$, and (4) $s \leq s^{\prime}$ implies $a s \leq a s^{\prime}$, for all $a, b \in A, s, s^{\prime} \in S$.

As pomonoids $S$ which we are dealing with in this paper, as monoids are semilattices, then they have so-called natural order such that, for $x, y \in$ $S, x$ is lower than or equal to $y$ if and only if $x=x y$. In what follows,

Received Received March 27, 2013.

2010 Mathematics Subject Classification. 06F05, 20M30.

Key words and phrases. Pomonoid, Rees factor, $S$-poset, property $\left(\mathrm{P}_{w}\right)$.

Research supported by Estonian Science Foundation Grant 8394.

http://dx.doi.org/10.12697/ACUTM.2013.17.09 
speaking about this natural order we use words lower or higher, and, to avoid ambiguity, speaking about the order $\leq$ in a pomonoid we use words smaller or larger.

In this paper we will be interested in the following property $\left(\mathrm{P}_{w}\right)$ which was introduced in [5].

An $S$-poset $A_{S}$ satisfies property $\left(\mathrm{P}_{w}\right)$ if, for all $a, b \in A$ and $s, t \in S$, $a s \leq b t$ implies $a \leq c u, c v \leq b$ for some $c \in A, u, v \in S$ with $u s \leq v t$.

Recall that an $S$-poset $A_{S}$ satisfies property (P) if, for all $a, b \in A$ and $s, t \in S$, as $\leq b t$ implies $a=c u, c v=b$ for some $c \in A, u, v \in S$ with $u s \leq v t$.

Clearly, property $(\mathrm{P})$ implies property $\left(\mathrm{P}_{w}\right)$. As it was shown in [5], property $\left(\mathrm{P}_{w}\right)$ still implies po-flatness.

A pomonoid $S$ is called weakly right reversible if for all $s, t \in S$ there exist $u, v \in S$ such that $u s \leq v t$.

Lemma 1 ([2]). Let $S$ be a pomonoid and $\Theta_{S}=\{\theta\}$ a one-element right $S$-poset. Then the following assertions are equivalent:

(a) $\Theta_{S}$ satisfies property $(\mathrm{P})$.

(b) $\Theta_{S}$ satisfies property $\left(\mathrm{P}_{w}\right)$.

(c) $S$ is weakly right reversible.

Let $\rho$ be an equivalence on an $S$-poset $A_{S}$. A $\rho$-chain is a finite sequence of elements $\left\{a, a_{1}, a_{1}^{\prime}, \ldots, a_{i}, a_{i}^{\prime}, \ldots, a_{n}, a_{n}^{\prime}, b\right\}$ of $A_{S}$ such that $a \leq a_{1}, a_{i} \rho a_{i}^{\prime}$ for $i=1, \ldots, n, a_{i}^{\prime} \leq a_{i+1}$ for $i=1, \ldots, n-1$ and $a_{n}^{\prime} \leq b$. A $\rho$-chain is said to be closed if $a=b$.

Let $A_{S}$ be an $S$-poset and $\rho$ be an $S$-act congruence. Then $\rho$ is an $S$-poset congruence if every closed $\rho$-chain is contained in a single congruence class of $\rho$.

An $S$-poset $A_{S}$ is called cyclic if $A_{S}=a S=\{a s \mid s \in S\}$ for some $a \in A_{S}$.

If $K_{S}$ is a convex right ideal of $S$, then there exists an $S$-poset congruence such that one of its classes is $K$ and all the others are singletons. The factor $S$-poset by this congruence is called the Rees factor $S$-poset of $S$ by $K$ and denoted $S / K$.

It was pointed out in [2] that, for $S$-posets as for $S$-acts, one has the following statement.

Lemma 2 ([2]). Let $K_{S}$ be a convex, proper right ideal of a pomonoid $S$. Then $S / K$ satisfies property $(\mathrm{P})$ if and only if $|K|=1$.

Property $\left(\mathrm{P}_{w}\right)$ was more closely investigated in [3] where pomonoids from a subclass of completely simple semigroups with adjoined identity all of whose cyclic (Rees factor) $S$-posets satisfy property $\left(\mathrm{P}_{w}\right)$ were characterized. Moreover, for the same class of pomonoids, necessary and sufficient 
conditions under which all Rees factor $S$-posets satisfying property $\left(\mathrm{P}_{w}\right)$ satisfy property $(\mathrm{P})$ were found.

Historically, the term homological classification of associative rings with an identity element is used as a general name for results which deduce properties of a ring from the properties of certain modules over it. One of the most important results in this line states that an associative ring with an identity element is regular (in the sense of von Neumann) if and only if all modules over it are flat.

Analogously, homological classification of monoids collects results which deduce properties of a monoid from the properties of acts over it. Different so-called flatness properties (projectivity, property (P), flatness, weak flatness, principal weak flatness, torsion freeness) have been widely used in the homological classification of monoids (see, for example, [4]). Although all acts over an arbitrary regular monoid are not flat, it is true that all acts over an inverse monoids are flat.

During recent years several articles on flatness properties of $S$-posets (projectivity, property $(\mathrm{P})$, property $\left(\mathrm{P}_{w}\right)$, po-flatness, flatness, weak po-flatness, weak flatness, principal weak po-flatness, principal weak flatness, po-torsion freeness, torsion freeness) have appeared. It has turned out that the situation here is very much different from that in the case of monoids and acts over them, and already on the level of posemilattices. So it follows from Theorem 22 of [2] that if all Rees factor $S$-posets over a pomonoid $S$, which is a semilattice with identity and whose pomonoid order coincides with its natural order, are flat then $S$ has at most two elements. At the same time, although property $\left(\mathrm{P}_{w}\right)$ is stronger than flatness, the results of this paper will show that all Rees factor acts over a posemilattice with an order different from the natural order may satisfy property $\left(\mathrm{P}_{w}\right)$ when $S$ has more than two elements, what is different from the unordered situation.

For more information on flatness properties of $S$-posets the reader is referred to [1].

\section{Results}

The following two results which were proved in [3] will be our main tool in what follows. It is easy to see that the first of them is just a reformulation of Lemma 5 of [3].

Lemma 3. Let $S$ be a pomonoid. The Rees factor $S$-poset $S / K$ by a convex, proper right ideal $K_{S}$ satisfies property $\left(\mathrm{P}_{w}\right)$ if and only if for any $k, l \in K$ one of the following three conditions is satisfied:
(a) $k \leq l$,
(b) there exist $p, q \in K$ such that $k \leq p l$ and $q<1$,
(c) there exist $p, q \in K$ such that $1<p$ and $q k \leq l$. 
Corollary 1. Let $S$ be a pomonoid and $K_{S}$ a convex, proper right ideal of $S$ such that one of the following three conditions is satisfied:

1) 1 is incomparable with all the elements of $K$,

2) $1>k$ for every $k \in K$,

3) $1<k$ for every $k \in K$.

If the Rees factor $S$-poset $S / K$ satisfies property $\left(\mathrm{P}_{w}\right)$, then $|K|=1$.

Since the $S$-poset $S_{S}$, being even free, satisfies property $(\mathrm{P})$, and since every semilattice, being commutative, is weakly right reversible, Lemma 1 implies the following statement.

Lemma 4. Let $S$ be a semilattice pomonoid. Then all Rees factor $S$ posets by a trivial convex ideal satisfy both property $(\mathrm{P})$ and property $\left(\mathrm{P}_{w}\right)$.

Since semilattice pomonoids $S,|S| \leq 2$, do not have nontrivial convex ideals, in what follows we will be interested only in semilattices $S,|S|>2$.

Notation. If $x, y \in S$ then

$$
[x, y]:=\{z \in S \mid x \leq z \leq y\},
$$

where $\leq$ is the pomonoid order.

It is easy to understand that if $I$ is a right ideal of a pomonoid $S$, then the smallest convex right ideal containing $I$ is $K=\{x \in S \mid s \leq x \leq t, s, t \in I\}$. Hence we have the following result.

Lemma 5. Let $S,|S|>2$, be an atomic semilattice pomonoid, e an atom and $K$ the smallest convex ideal of $S$ containing $e$. If $0<e$, then $K=[0, e]$, if $e<0$, then $K=[e, 0]$, and if $e$ and 0 are incomparable, then $K=\{0, e\}$.

Our further aim is to characterize atomic semilattice pomonoids $S$ all of whose Rees $S$-posets by a convex ideal satisfy property $\left(\mathrm{P}_{w}\right)$. We start with pomonoids in which 1 and 0 are comparable.

Theorem 1. Let $S,|S|>2$, be an atomic semilattice pomonoid such that 1 and 0 are comparable. Then the following assertions are equivalent:

(i) All Rees factor $S$-posets satisfy property $(P)$.

(ii) All Rees factor $S$-posets satisfy property $\left(\mathrm{P}_{w}\right)$.

(iii) $S$ has only one atom e and either $S=[0, e]$ or $S=[e, 0]$.

Proof. (i) $\Rightarrow$ (ii) is obvious since property $(\mathrm{P})$ implies property $\left(\mathrm{P}_{w}\right)$.

(ii) $\Rightarrow$ (iii). Assume that all Rees factor $S$-posets satisfy property $\left(\mathrm{P}_{w}\right)$. Let $e$ be an atom in the semilattice $S$.

Suppose first that $0<1$. Multiplying the inequality $0<1$ by $e$, we obtain $0<e$. Let $K$ be the smallest convex ideal of $S$ containing $e$. Then $K=[0, e]$ by Lemma 5 . The Rees factor $S$-poset $S / K$ satisfies property $\left(\mathrm{P}_{w}\right)$ by assumption. Suppose that $K$ is a proper ideal. Then it follows from 
Lemma 3 that for $e$ and 0 in $K$ there exist $p, q \in K$ such that either condition (b) or condition (c) of that lemma is satisfied. This cannot be condition (c) since in this case $1<p, p \in K$, which implies $1<e$, and thus $1 \in K$. Then $K=S$, a contradiction. Hence condition (b) of Lemma 3 is satisfied. But then $e \leq p 0=0$, a contradiction. Hence $K$ cannot be a proper ideal. But then $S=K=[0, e]$.

Moreover, $S=[0, e]$ implies that $e$ is the only atom in the semilattice $S$.

A dual argument gives us that if $1<0$ then $S=[e, 0]$ which also implies that $e$ is the only atom in the semilattice $S$.

(iii) $\Rightarrow$ (i). Assume that $S$ has only one atom $e$ and $S=[0, e]$ or $S=[e, 0]$. Then every bigger than one-element convex ideal of $S$, containing necessarily $e$ and 0 and so also 1 , coincides with $S$. In other words, $S$ does not contain non-trivial convex ideals and hence all Rees factor $S$-posets satisfy property (P) by Lemma 4.

Now we turn to pomonoids where 1 and 0 are incomparable. We continue with semilattices having at least two atoms.

Theorem 2. Let $S,|S|>2$, be an atomic semilattice pomonoid such that 1 and 0 are incomparable and the semilattice $S$ contains at least two atoms. Then all Rees factor $S$-posets by a convex ideal satisfy property $\left(\mathrm{P}_{w}\right)$ if and only if the semilattice $S$ contains exactly two atoms $e$ and $f$ such that either $f<1<e$ or $e<1<f$ in the pomonoid $S$.

Proof. For necessity assume that all Rees factor $S$-posets by a convex ideal satisfy property $\left(\mathrm{P}_{w}\right)$. Suppose that $e$ and $f$ are different atoms in the semilattice $S$. Let $K$ be the smallest convex ideal of $S$ containing $e$. Then $K$ is a proper convex ideal. Indeed, if $K=S$, then Lemma 5 implies that $0 \leq 1 \leq e$ or $e \leq 1 \leq 0$ and thus 0 and 1 are comparable. Now the Rees factor $S$-poset $S / K$ satisfies property $\left(\mathrm{P}_{w}\right)$ by assumption and it follows from Corollary 1 that 1 has to be comparable with an element from $K$. As 1 is incomparable with 0 it follows easily from Lemma 5 that 1 has to be comparable with $e$. By the same reason 1 has to be comparable with $f$.

If $e<1$ and $f<1$, then, multiplying the inequality $e<1$ by $f$, we get $0<f$ which together with $f<1$ gives $0<1$, a contradiction. If $1<e$ and $1<f$, then, multiplying the inequality $1<e$ by $f$, we get $f<0$ which together with $1<f$ gives $1<0$, a contradiction.

If $1<e$ and $f<1$, then $f<1<e$. This implies $f s \leq s \leq e s$ for every $s \in S$. If $g \in S$ is an atom in the semilattice $S$ such that $g \neq e$ and $g \neq f$, then $0=f g \leq g \leq e g=0$ and thus $g=0$, a contradiction. Hence $e$ and $f$ are the only atoms in the semilattice $S$.

If $e<1$ and $1<f$, then, of course, $e<1<f$ and in this case $e$ and $f$ are also the only atoms of $S$. 
For sufficiency assume that $S$ has two atoms $e$ and $f$ such that $f<1<e$. Notice first that, multiplying the inequality $1<e$ by $f$, we get $f<0$. Similarly, from $f<1$ we get $0<e$.

Notice, moreover, that since $S$ is an atomic semilattice with two atoms $e$ and $f$, for an element different from atoms and 0 there are three options: to be higher than both $e$ and $f$, or to be higher than $e$ and not higher than $f$, or to be higher than $f$ but not higher than $e$.

Let $K$ be a convex ideal of the pomonoid $S$. If $K$ contains an element which is higher than both $e$ and $f$ in the semilattice $S$, then $K$, containing $e$ and $f$ and being convex, contains also 1 and thus $K=S$. Hence $S / K$ satisfies property $\left(\mathrm{P}_{w}\right)$ by Lemma 4 .

Let $y$ be an element higher than $e$ but not higher than $f$ in the semilattice $S$. Then, multiplying the inequality $f<1$ by $y$, we get $0<y$. Multiplying the inequality $1<e$ by $y$, we get $y<e$. This means that $y \in K=[0, e]$. By Lemma $5 K$ is the smallest convex ideal containing $e$. Now it is clear that $K$ is the smallest convex ideal containing $y$ for every $y$ higher than or equal to $e$ but not higher than $f$. Moreover, $K$ is the only nontrivial convex ideal containing such $y$. Indeed, otherwise we would have $f \in K$ and thus $K=S$.

Suppose $k, l \in K$. Then, taking $q=0$ and $p=e$, we have $1<p$ and $q k=0 k=0 \leq l$. This means that condition (c) of Lemma 3 is satisfied and thus $S / K$ satisfies property $\left(\mathrm{P}_{w}\right)$.

Finally, a dual argument gives us that every element $y$ which is higher than $f$ but not higher than $e$ in the semilattice $S$ belongs to $K=[f, 0]$ and that $K$ is the only nontrivial convex ideal containing such $y$. For arbitrary elements $k, l \in K$ condition (b) of Lemma 3 holds and thus $S / K$ satisfies property $\left(\mathrm{P}_{w}\right)$.

Lemma 6. Let $S,|S|>2$, be an atomic semilattice pomonoid such that 1 and 0 are incomparable and the semilattice $S$ contains one atom e. If all Rees factor $S$-posets by a convex ideal satisfy property $\left(\mathrm{P}_{w}\right)$, then $e$ is the smallest element or the largest element in the pomonoid $S$.

Proof. Assume that all Rees factor $S$-posets by a convex ideal satisfy property $\left(\mathrm{P}_{w}\right)$. Let $K$ be the smallest convex ideal of $S$ containing $e$. Then $K$ is a proper convex ideal. Indeed, if $K=S$, then Lemma 5 implies that $0 \leq 1 \leq e$ or $e \leq 1 \leq 0$ and thus 0 and 1 are comparable. Now the Rees factor $S$-poset $S / K$ satisfies property $\left(\mathrm{P}_{w}\right)$ by assumption and it follows from Corollary 1 that 1 has to be comparable with an element from $K$. As 1 is incomparable with 0 it follows easily from Lemma 5 that 1 has to be comparable with $e$.

Let, first, $e<1$. As the Rees factor $S$-poset $S / K$ satisfies property $\left(\mathrm{P}_{w}\right)$ it follows from Lemma 3 that for $e$ and 0 in $K$ one of the three conditions of that lemma is satisfied. Since $e \not \leq 0$, conditions (a) and (b) of Lemma 3 cannot be satisfied. Hence condition (c) is satisfied. But then there exist 
$p, q, \in K$ such that $1<p$ and $q e \leq 0$. By Lemma $5, K=[0, e]$, or $K=\{e, 0\}$, or $K=[e, 0]$. The first two of these equalities together with $1<p$ imply $1<e$, a contradiction. Hence $e<0$. If $x \in S$ is higher than $e$ in the semilattice $S$, then, multiplying the inequality $e<1$ by $x$, we get $e<x$. Thus $e$ is the smallest element in the pomonoid $S$.

If $1<e$, then a dual argument gives us that $e$ is the largest element in the pomonoid $S$.

The following lemma is a direct corollary of Lemma 3 .

Lemma 7. Let $S,|S|>2$, be a semilattice pomonoid. If 1 is a maximal element, then the Rees factor $S$-poset $S / K$ by a convex, proper ideal $K$ satisfies property $\left(\mathrm{P}_{w}\right)$ if and only if for any $k, l \in K$ either $k \leq l$ or there exist $p, q \in K$ such that $k \leq p l$ and $q<1$.

If 1 is a minimal element, then the Rees factor $S$-poset $S / K$ by a convex, proper ideal $K$ satisfies property $\left(\mathrm{P}_{w}\right)$ if and only if for any $k, l \in K$ either $k \leq l$ or there exist $p, q \in K$ such that $1<p$ and $q k \leq l$.

Theorem 3. Let $S,|S|>2$, be an atomic semilattice pomonoid such that 1 is a maximal (minimal) element, 1 and 0 are incomparable and the semilattice $S$ contains one atom e. Then all Rees factor $S$-posets by a convex ideal satisfy property $\left(\mathrm{P}_{w}\right)$ if and only if $e$ is the smallest (largest) element and $x \leq 0$ for every $x \in S, x \neq 1 \quad(0 \leq x$ for every $x \in S, x \neq 1)$.

Proof. For necessity assume first that 1 is a maximal element and that all Rees factor $S$-posets by a convex ideal satisfy property $\left(\mathrm{P}_{w}\right)$. Since 1 is a maximal element, $e$ cannot be the largest element and hence, by Lemma $6, e$ is the smallest element of the pomonoid $S$. Take $x \in S, x \neq 1$, and let $K$ be the smallest convex ideal of $S$ containing $x$. Since 1 is a maximal element, $K$ is a proper ideal. Now the Rees factor $S$-poset $S / K$ satisfies property $\left(\mathrm{P}_{w}\right)$ by assumption and it follows from Lemma 7 that for $x$ and 0 in $K$ there exists $p \in K$ such that $x \leq p 0=0$. Dually, if 1 is a minimal element, then one gets that $e$ is the largest element and $0 \leq x$ for every $x \in S, x \neq 1$.

For sufficiency suppose first that 1 is a maximal element, $e$ is the smallest element and $y \leq 0$ for every $y \in S, y \neq 1$. This means that $y \in K=[e, 0]$ for every element $y \in S, y \neq 1$. By Lemma $5, K$ is the smallest convex ideal containing $e$. Now it is clear that $K$ is the smallest convex ideal containing every $y \in S, y \neq 1$. Moreover, $K$ is the only nontrivial convex ideal. Suppose $k, l \in K$. Then, taking $p=0$ and $q=e$, we have $k \leq 0=0 l=p l$ and $q<1$. This means, by Lemma 7 , that $S / K$ satisfies property $\left(\mathrm{P}_{w}\right)$.

Dually, one can show that if 1 is a minimal element, $e$ is the largest element and $0 \leq x$ for every $x \in S, x \neq 1$, then all Rees factor $S$-posets by a convex ideal satisfy property $\left(\mathrm{P}_{w}\right)$. 
Lemma 8. Let $S,|S|>2$, be an atomic semilattice pomonoid such that 1 is neither a maximal, nor a minimal element and the semilattice $S$ satisfies the minimum condition. Then there exists the lowest element among the elements $x \in S$ such that $1<x(x<1)$.

Proof. Suppose 1 is not a maximal element. Then there exist elements $x \in S$ such that $1<x$. As the semilattice $S$ satisfies the minimum condition there are minimal elements among such elements. Suppose $y$ and $z$ are two minimal elements among the elements $x \in S$ such that $1<x$. Then, clearly, $y$ and $z$ are incomparable in the semilattice $S$. Multiplying the inequality $1<z$ by $y$, we get $y<y z$. The last inequality together with $1<y$ gives us $1<y z$ which is impossible since $y z$ is lower than $y$. Hence there exists the lowest element among the elements $x \in S$ such that $1<x$.

A dual argument can be used to show that if 1 is not a minimal element, then there exists the lowest element among the elements $x \in S$ such that $x<1$.

Remark 1. In the last remaining case if $S,|S|>2$, is an atomic semilattice pomonoid such that 1 is neither a maximal, nor a minimal element, 1 and 0 are incomparable and the semilattice $S$ contains one atom, then we are able to obtain a characterization of pomonoids all of whose Rees factor $S$-posets satisfy property $\left(\mathrm{P}_{w}\right)$ only for semilattices satisfying the minimum condition. Clearly, finite semilattices are still included.

Theorem 4. Let $S,|S|>2$, be an atomic semilattice pomonoid such that 1 is neither a maximal, nor a minimal element, 1 and 0 are incomparable, the semilattice $S$ satisfies the minimum condition and contains only one atom $e$. Then all Rees factor $S$-posets by a convex ideal satisfy property $\left(\mathrm{P}_{w}\right)$ if and only if

(i) $e$ is the largest element and $0 \leq x$ for every $x \in S, x$ is lower than or incomparable with $d$, where $d$ is the lowest element among the elements $x \in S$ such that $x<1$, or

(ii) $e$ is the smallest element and $x \leq 0$ for every $x \in S, x$ is lower than or incomparable with $c$, where $c$ is the lowest element among the elements $x \in S$ such that $1<x$.

Proof. For necessity assume that all Rees factor $S$-posets by a convex ideal satisfy property $\left(\mathrm{P}_{w}\right)$. Then it follows from Lemma 6 , that $e$ is either the largest element or the smallest element.

Suppose, first, that $e$ is the largest element. By Lemma 8 there exists $d \in S$, the lowest element among the elements $x \in S$ such that $x<1$. Since $e$ is the largest element, $d \neq e$. Let now $y \in S$ be an element lower than $d$ or incomparable with $d$ and let $K$ be the smallest convex ideal containing $y$. If $K=S$, then there exists $s \in S$ such that $y s<1$ which is impossible since $y s$ is lower than $d$ or incomparable with $d$. Hence $K$ is a proper convex ideal. 
Now the Rees factor $S$-poset $S / K$ satisfies property $\left(\mathrm{P}_{w}\right)$ by assumption and it follows from Lemma 3 that for 0 and $y$ in $K$ one of the three conditions of this lemma is satisfied. This cannot be condition (b) since otherwise we would have $q<1, q \in K$. But then there exists $t \in S$ such that $y t \leq q$. This inequality together with $q<1$ implies $y t<1$ which is impossible since $y t$ is lower than $d$ or incomparable with $d$. Suppose condition (c) of Lemma 3 is satisfied. Then there exists $q \in K$ such that $q 0 \leq y$ which implies $0 \leq y$. Hence condition (i) holds.

A dual argument gives us that if $e$ is the smallest element then condition (ii) holds.

For sufficiency assume, first, that condition (i) holds. Let $d \in S$ be the lowest element among the elements $x \in S$ such that $x<1$. Let $K$ be a convex ideal of pomonoid $S$. If $K$ contains an element which is higher than or equal to $d$, then $K$, containing $d$ and $e$ and being convex, contains also 1 and thus $K=S$. Hence $S / K$ satisfies property $\left(\mathrm{P}_{w}\right)$ by Lemma 4 .

Let $y$ be an element lower than $d$ or incomparable with $d$ in the semilattice $S$. Then $y \in K=[0, e]$ by assumption. By Lemma $5 K$ is the smallest convex ideal containing $e$. Now it is clear that $K$ is the smallest convex ideal containing $y$ for every $y$ lower than $d$ or incomparable with $d$. Moreover, $K$ is the only nontrivial convex ideal containing such $y$. Indeed, otherwise we would have $d \in K$ and thus $K=S$.

Suppose $k, l \in K$. Then, taking $p=e$ and $q=0$, we have $1<e=p$ and $q k=0 k=0 \leq l$. This means that condition (c) of Lemma 3 is satisfied and thus $S / K$ satisfies property $\left(\mathrm{P}_{w}\right)$.

A dual argument gives us that if condition (ii) holds then all Rees factor $S$-posets by a convex ideal satisfy property $\left(\mathrm{P}_{w}\right)$.

Acknowledgement. The author is grateful to the referee for a number of useful suggestions.

\section{References}

[1] S. Bulman-Fleming, Flatness properties of S-posets: an overview, in: Proceedings of the International Conference on Semigroups, Acts and Categories with Applications to Graphs, Mathematics Studies 3, Estonian Mathematical Society, Tartu, 2008, pp. $28-40$.

[2] S. Bulman-Fleming, D. Gutermuth, A. Gilmour, and M. Kilp, Flatness properties of S-posets, Comm. Algebra 34, (2006), 1291-1317.

[3] M. Kilp, On the homological classification of pomonoids by properties of cyclic S-posets, Semigroup Forum 86 (2013), 592-602.

[4] M. Kilp, U. Knauer, and A. V. Mikhalev, Monoids, Acts and Categories, de Gruyter Expositions in Mathematics 29, Walter de Gruyter, Berlin, 2000.

[5] X. Shi, Strongly flat and po-flat S-posets, Comm. Algebra 33 (2005), 4515-4531.

Institute of Mathematics, University of TARTu, 50409 Tartu, Estonia

E-mail address: mati.kilp@ut.ee 